\title{
Functionally-Graded Polymeric Graft Substitutes and Scaffolds for Tissue Engineering can be Fabricated via Various Extrusion Methods
}

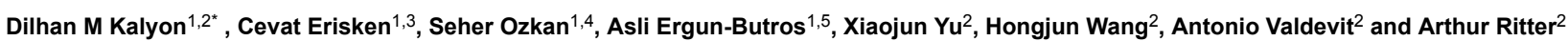 \\ ${ }^{1}$ Chemical Engineering and Materials Science, Stevens Institute of Technology, USA \\ ${ }^{2}$ Chemistry, Chemical Biology and Biomedical Engineering Department, Stevens Institute of Technology, USA \\ ${ }^{3}$ Tissue Engineering and Regenerative Medicine Laboratory, Columbia University Medical Center, USA \\ ${ }^{4}$ Ashland Specialty Ingredients, Ashland Inc., Bridgewater, USA \\ 524 M Company, Cambridge, MA
}

*Corresponding author: Dilhan M Kalyon, Chemistry, Chemical Biology and Biomedical Engineering Department, Stevens Institute of Technology, Hoboken, NJ, USA, Tel: 201-216-8225; Email: dkalyon@stevens.edu

Received date: March 25, 2014, Accepted date: March 27, 2014, Published date: March 29, 2014

Copyright: (c) 2014 Kalyon DM, et al. This is an open-access article distributed under the terms of the Creative Commons Attribution License, which permits unrestricted use, distribution, and reproduction in any medium, provided the original author and source are credited.

\section{Abstract}

The design and fabrication of bioresorbable polymeric graft substitutes and porous scaffolds for regenerative medicine are challenged by the complexities in structure and composition found in native tissues. The mimicking of complex gradations found in native tissues requires correspondingly complex gradations in bone graft substitutes and tissue engineering scaffolds. Extrusion based processing methods offer significant advantages for the fabrication of scaffolds and polymeric graft substitutes functionally-graded in various directions for porosity, composition as well as distributions of other tissue relevant properties.

\section{Challenges of Regenerative Medicine}

The design and fabrication of bioresorbable polymeric graft substitutes and porous scaffolds for regenerative medicine are challenged by the complex structural and compositional gradations found in human tissues [1-6] and tissue-tissue transitions such as the interfaces between cartilage and bone, ligament and bone, and tendon and bone. For example, it would be desirable for the bone graft substitutes used in the repair of critical sized defects in long bones like femur and tibia to accommodate changes in porosities and moduli along their transverse and axial directions. The mimicking of complex gradations found in native tissues requires correspondingly complex gradations in bone graft substitutes and tissue engineering scaffolds. Generally, efforts to generate graft substitutes and scaffolds which exhibit tailored three-dimensional distributions in composition, structure and properties are constrained by the myriad shortcomings of conventional scaffold fabrication methodologies. Examples of such efforts [1-6] include the multilayered scaffolds that were fabricated via electrospinning of separate meshes and pressing them under hydraulic pressure, layer-by-layer casting, freeze-drying, phase separation, and rapid prototyping techniques, including fused deposition modeling, 3D printing, selective laser sintering, and stereo lithography. Among these, the 3-D printing method offers significant promise but is handicapped by the unavailability of the mixing and dispersion capability, and its typical requirement of high temperature processing for some polymers that could be detrimental for biological additives. Thus, additional methods that can process a wide family of polymers and additives (including bioactives) and that would allow the reproducible and industrially-scalable grading of graft substitutes and tissue engineering scaffolds for a wide range of compositions, porosities and mechanical properties are needed.

Extrusion based fabrication methods offer significant advantages for the fabrication of graded scaffolds and polymeric graft substitutes as described in the following. Such methods have been largely overlooked in spite of their advantages for generating graded structures over conventional methods of fabrication.

\section{Processing and Shaping of Polymer Melts and Solution and Polymeric Compounds via Extrusion Methods}

Extruders can be of the single or the twin screw extruder type with the twin screw extruders further subdivided into co-rotating (rotation directions of the two screws are similar) or counter-rotating. Twin screw extruders can also be of the fully-intermeshing or nonintermeshing, i.e., tangential types. Twin screw extruders are preferred over the single screw extruders due to their superior mixing capabilities and greater versatility. Extruders allow multiple ingredients to be fed, polymers to be molten, various ingredients to be mixed, air/ gases to be removed and the mixtures to be pressurized to be forced through dies for being shaped into extrudates. The screw elements can have different functionalities, i.e., regular-flighted conveying screws and lenticular elements, i.e., the kneading disks. The kneading disks provide chaotic mixing dynamics as well as generate a dispersive mixing capability, to enable the break-up of particle agglomerates, i.e., a capability not found in conventional scaffold fabrication methods [7, 8]. A die is attached to the exit of the extruder to generate the desired shape. Instead of a die an electrospinning head can also be utilized. To generate porosity either porogens like dissolvable polymers or salts or foaming methods based on chemical and physical blowing agents, including supercritical fluid technologies [9], can be utilized.

The extrusion process can provide functionally graded extrudates to serve as graded scaffolds or polymeric implants simply via timedependent changes in the operating conditions and feed rates during the process. Rendering the input rates and process conditions cyclic gives rise to corresponding cyclic changes in the composition, porosity and physical properties either as a function of the axial or the transverse to flow direction or as functions of both axial and transverse to flow directions. 


\section{Extrusion Methods for Generating Functionally Graded Polymeric Implants and Scaffolds}

Various extrusion based fabrication methods have been demonstrated for the manufacture of graded extrudates [9-20]. The principal methods are:

\section{Extrusion using co-extrusion dies [10-14]}

Hybrid extrusion and spiral winding method $[15,16]$

\section{Hybrid twin screw extrusion and electrospinning method [17-20]}

All three methods are amenable to industrial scale-up and generate scaffolds which are reproducible in geometry and properties. All three methods can be used with and without solvents (dry versus wet extrusion methods). All three were applied in the area of interface tissue engineering, targeting regenerative medicine for bone and cartilage repair and regeneration [10-20]. They possess the ability to introduce various ingredients of the formulation/s in a time-dependent fashion into the extruder for the manufacture of spatially (radially and axially) graded, i.e., "functionally-graded" scaffolds $[14,16,18,20]$. The availability of such graded scaffolds has allowed the control of the spatial distributions of bioactives, porosity, pore sizes, mechanical properties. Typically various bioresorbable polymeric resins, including poly (glycolic acid) (PGA), poly (lactic acid) (PLA), and poly (caprolactone) (PCL), were compounded in the extruder with one or more porogens, i.e., generally dissolvable salts and polymers, or with physical (typically supercritical $\mathrm{CO}_{2}$ ) or chemical blowing agents to induce porosity with interconnected pores. Various bioactives including nanoparticles, drugs, and proteins could also be incorporated. Mathematical models of the extrusion processes are very useful to determine the geometry and conditions necessary to give rise to the desired grading of the polymeric implant and scaffold [21-23].

In the co-extrusion process a co-extrusion die was used to fabricate extrudates with multiple layers and cage/core structures, i.e., polymeric bone graft substitutes, to address the challenge of the repair and regeneration of bone defects [10-14]. The extrudates were both radially and axially-graded to generate multi-zonal bone graft substitutes fabricated from poly(caprolactone), PCL, and PCL bio-composites with osteoconductive particles, i.e., hydroxyapatite, HA, and $\beta$ tricalcium phosphate, TCP. Such graft substitutes would provide a greater degree of freedom to the orthopedic surgeon especially for repair of critically-sized bone defects. The modulus of the graft substitute could be tailored in the axial direction upon the systematic variation of the HA/TCP concentration while in the radial direction the bone graft substitute consisted of an outer layer with high stiffness, encapsulating a softer core with greater porosity. The bone graft substitutes were shown to be biocompatible via in vitro culturing of human bone marrow-derived stromal cells, BMSCs, followed by the analysis of cell proliferation and differentiation [14]. The characterization of the tissue constructs included the enzymatic alkaline phosphates (ALP) activity, $\mu$-CT imaging and PCR analysis involving the expressions of bone-related markers, i.e. Runx2, ALP, collagen type I, osteopontin and osteocalcin, overall demonstrating the differentiation of BMSCs via osteogenic lineage and formation of mineralized bone tissue [14]

The twin screw extrusion and spiral winding, TSESW, process consists of the integration of the twin screw extrusion process with a modified filament winding method (designated here as "spiral winding") $[15,16]$. The extrudate emerging from the die is wound around a mandrel that concomitantly rotates and translates sideways creating a helical and spiral trajectory for the fabrication of cylindrical extrudates $[15,16]$. Scaffolds with a wide range of sizes and thicknesses, and radial and axial distributions of pore size and porosity could be developed primarily addressing relatively large critical bone defects [16]. In one application annular scaffolds were fabricated from PCL incorporated with nanoparticles of $\beta$-TCP and the extrudates were functionally graded in terms of pore size and composition. The ability to change the porosity, pore size, and $\beta$-TCP nanoparticle concentration in each layer enabled the investigation of the effects of introducing porosity, pore size, and composition distributions on compressive properties. Various proteins could be incorporated into the scaffolds without denaturing [15]. Using human fetal osteoblast cells it was demonstrated that the resulting functionally graded scaffolds are biocompatible with favorable cell adhesion, penetration, and differentiation $[15,16]$.

The third method, i.e., the integration of the extrusion process with electrospinning, provides nanofibrous meshes [17-20] which can be incorporated with various types of bioactives to target the generation of especially interface tissues, for example the bone and cartilage interface $[19,20]$. A demonstration using PCL involved altering the concentration distributions of two bioactive agents concomitantly (one increasing, whereas the other decreasing monotonically) in between the two sides of a nanofibrous scaffold, i.e., the gradients of insulin, a stimulator of chondrogenic differentiation, and $\beta$-glycerophosphate $(\beta$ GP), for mineralization [20]. The graded mesh was seeded with human adipose-derived stromal cells and cultured over eight weeks. The resulting tissue constructs revealed selective differentiation of the stromal cells toward chondrogenic lineage and mineralization as functions of position resulting from the corresponding concentrations of insulin and $\beta$-GP. Chondrogenic differentiation of the stem cells increased at insulin-rich locations and mineralization increased at $\beta$ GP-rich locations [20].

Combinations of such extrusion based methods are also possible. For example, the electrospinning process can be married to the spiral winding process for the fabrication of multi-featured scaffolds which consist of spiral wound shells surrounding electrospun cores [24]. Such a combination was shown to be suitable for fabricating multiscale scaffolds for achieving vascularized osteon-like structures [24].

In summary extrusion-based processing methods present versatile technologies that are capable of creating complex gradations in graft substitutes and tissue engineering scaffolds. They should be considered for utilization as either stand-alone or as complementary technologies in various demanding tissue engineering applications.

\section{References}

1. W Pompe, H Worch, M. Epple, W. Friess, M. Gelinsky, et al. (2003) Functionally graded materials for biomedical applications. Mater SciEng 362: 40-60.

2. AG Mikos, SW Herring, P Ochareon, J Elisseeff, HH Lu, et al. (2006) Engineering complex tissues. Tissue Eng 12: 3307-3339.

3. Leong KF, Chua CK, Sudarmadji N, Yeong WY (2008) Engineering functionally graded tissue engineering scaffolds. J MechBehav Biomed Mater 1: 140-152.

4. Lu HH, Subramony SD, Boushell MK, Zhang X (2010) Tissue engineering strategies for the regeneration of orthopedic interfaces. Ann Biomed Eng 38: 2142-2154.

5. D Puppi, F Chiellini, AM Piras, E Chiellini, (2010) Polymeric materials for bone and cartilage repair. ProgPolymSci 35: 403-440. 
Citation: Dilhan M Kalyon, Cevat Erisken, Seher Ozkan, Asli Ergun-Butros, Xiaojun Yu, Hongjun Wang, Antonio Valdevit and Arthur Ritter (2014) Functionally-Graded Polymeric Graft Substitutes and Scaffolds for Tissue Engineering can be Fabricated via Various Extrusion Methods. J Tissue Sci Eng 5: e128. doi:10.4172/2157-7552.1000e128

Page 3 of 3

6. Seidi A, Ramalingam M, Elloumi-Hannachi I, Ostrovidov S, Khademhosseini A (2011) Gradient biomaterials for soft-to-hard interface tissue engineering. ActaBiomater 7: 1441-1451.

7. A Lawal, DM Kalyon (1995) Mechanisms of Mixing in Single and Corotating Twin Screw Extruders. Polymer Engineering and Science 35: 1325-1338.

8. A Lawal, DM Kalyon (1995) Simulation of the Intensity of Segregation Distributions Using Three-Dimensional FEM Analyses: Applications to Co-rotating Twin Screw Extruders. Journal of Applied Polymer Science 58: 1501-1507.

9. S Aktas, H Gevgilili, I Kucuk, A Sunol, DM Kalyon (2013) Extrusion of poly(ether imide) foams using pressurized CO2: Effects of imposition of supercritical conditions and nanosilica modifiers. Polymer Engineering and Science DOI: 10.1002/pen.23753.

10. A Ergun, R Chung, D Ward, A Valdevit, A Ritter, et al. (2012) Unitary bioresorbable cage/core bone graft substitutes for spinal arthrodesis coextruded from polycaprolactonebiocomposites. Ann Biomed Eng 40: 1073-1087.

11. Ergun A, Yu X, Valdevit A, Ritter A, Kalyon DM (2011) in vitro analysis and mechanical properties of twin screw extruded single-layered and coextruded multilayered poly(caprolactone) scaffolds seeded with human fetal osteoblasts for bone tissue engineering. J Biomed Mater Res A 99: 354-366.

12. A Ergun, $X$ Yu, A Valdevit, A Ritter, DM Kalyon (2011) In vitro analysis and mechanical properties of twin screw extruded single-layered and coextruded multi-layered poly(caprolactone) scaffolds seeded with human fetal osteoblasts for bone tissue engineering. J Biomed Mater Res 99: 354-366.

13. A Ergun, DM Kalyon, A Valdevit, A Ritter (2011) Compressive fatigue behavior of osteoblast seeded tissue constructs of poly(caprolactone) multilayered scaffolds for bone graft substitute applications. Orthopaedic Research Society Transactions 36: 1850.

14. A Ergun, X Yu, A Valdevit, A Ritter, DM Kalyon (2012) Radially and axially-graded multi-zonal scaffolds targeting critical-sized bone defects from polycaprolactone/hydroxyapatite/tricalcium phosphate. Tissue Eng Part A 18: 2426-2436.

15. S Ozkan, DM Kalyon, X Yu, C McKelvey, M Lowinger (2009) Multifunctional protein-encapsulated polycaprolactone scaffolds:
Fabrication and in vitro assessment for tissue engineering. Biomaterials 30: 4336-4347.

16. S Ozkan, DM Kalyon, X Yu (2010) Functionally graded beta-TCP/PCL nanocomposite scaffolds: in vitro evaluation with human fetal osteoblast cells. J Biomed Mater Res A 92: 1007-1018.

17. Erisken C, Kalyon DM, Wang H (2008) A hybrid twin screw extrusion/ electrospinning method to process nanoparticle-incorporated electrospun nanofibres. Nanotechnology 19: 165302.

18. C Erisken, DM Kalyon, H Wang (2008) Functionally and continuously graded electrospunpolycaprolactone and ß-tricalcium phosphate nanocomposites for interface tissue engineering applications. Biomaterials 29: 4065-4073.

19. Erisken C, Kalyon DM, Wang H (2010) Viscoelastic and biomechanical properties of osteochondral tissue constructs generated from graded polycaprolactone and beta-tricalcium phosphate composites. Journal of Biomechanical Engineering 132: 091013.

20. C Erisken, DM Kalyon, C Ornek-Ballanco, H Wang, J Xu (2011) Osteochondral tissue formation through adipose-derived stem cell differentiation using biomimetic tissue scaffolds with graded stimulator concentrations. Tissue Eng Part A 17: 1239-1252.

21. M Malik, DM Kalyon (2005) Three-dimensional Finite Element Simulation of Processing of Generalized Newtonian Fluids in Counter-rotating and Tangential Twin Screw Extruder and Die Combination. International Polymer Processing 20: 398- 409.

22. DM Kalyon, M Malik (2007) An integrated approach for numerical analysis of coupled flow and heat transfer in co-rotating twin screw extruders. International Polymer Processing 22: 293-302.

23. M Malik, DM Kalyon, JC Golba Jr (2014) Simulation of co-rotating twin screw extrusion process subject to pressure-dependent wall slip at barrel and screw surfaces: 3D FEM Analysis for combinations of forward- and reverse-conveying screw elements. International Polymer Processing, 29: 51-62.

24. Chen X, Ergun A, Gevgilili H, Ozkan S, Kalyon DM, et al. (2013) Shell-core bi-layered scaffolds for engineering of vascularized osteon-like structures. Biomaterials 34: 8203-8212. 